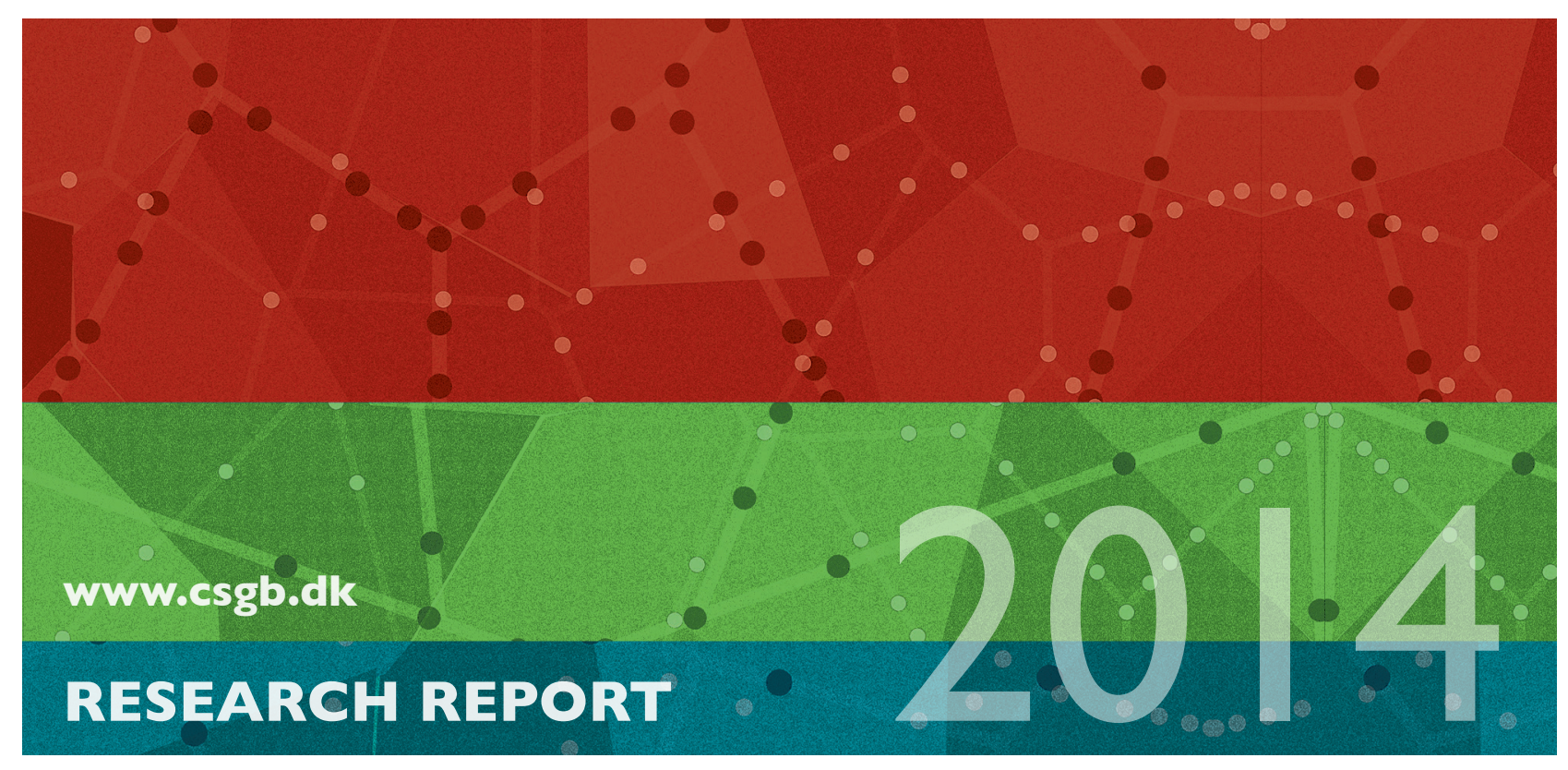

Anders Rønn-Nielsen and Eva B. Vedel Jensen

Tail asymptotics for the supremum of an infinitely divisible field with convolution equivalent Lévy measure

No. 09, May 2014 


\title{
Tail asymptotics for the supremum of an infinitely divisible field with convolution equivalent Lévy measure
}

\author{
Anders Rønn-Nielsen ${ }^{1}$ and Eva B. Vedel Jensen ${ }^{2}$ \\ ${ }^{1}$ Department of Mathematical Sciences, University of Copenhagen \\ ${ }^{2}$ Department of Mathematics, Aarhus University
}

\begin{abstract}
We consider a continuous, infinitely divisible random field in $\mathbb{R}^{d}$ given as an integral of a kernel function with respect to a Lévy basis with convolution equivalent Lévy measure. For a large class of such random fields we compute the asymptotic probability that the supremum of the field exceeds the level $x$ as $x \rightarrow \infty$. Our main result is that the asymptotic probability is equivalent to the right tail of the underlying Lévy measure.
\end{abstract}

Keywords: Asymptotic supremum; convolution equivalence; infinite divisibility; Lévy-based modelling

\section{Introduction}

In the present paper we investigate the extremal behaviour of a field $\left(X_{t}\right)_{t \in B}$ defined by

$$
X_{t}=\int_{\mathbb{R}^{d}} f(|t-s|) M(\mathrm{~d} s),
$$

where $M$ is an infinitely divisible, independently scattered random measure on $\mathbb{R}^{d}$, $f$ is some kernel function, and $B$ is a compact index set. We will assume that the Lévy measure of the random measure $M$ has a convolution equivalent right tail $([11,12,21])$.

Under some further regularity conditions we derive in the present paper for a random field (1.1) the very useful result that the asymptotic behaviour of the supremum of $X_{t}, t \in B$, has a tail that is equivalent to the tail of the underlying Lévy measure. More precisely under the assumption that the underlying Lévy measure of $M$ has a tail that is asymptotically equivalent to $x^{-\delta} \exp (-\beta x), \beta>0, \delta>1$, we show that

$$
P\left(\sup _{t \in B} X_{t}>x\right) \sim C x^{-\delta} \exp (-\beta x) E \exp \left(\beta X_{t_{0}}\right) m_{d}(B)
$$

Corresponding author: Anders Rønn-Nielsen, arnielsen@math.ku.dk 
as $x \rightarrow \infty$, where $C$ is a known constant and $m_{d}(B)$ is the Lebesgue measure of $B$. Measures with a tail asymptotically equivalent to $x^{-\delta} \exp (-\beta x)$ are indeed convolution equivalent ([21, Lemma 2.3]) and cover the important cases of an inverse Gaussian and a normal inverse Gaussian (NIG) basis, respectively, see Section 2 below.

Lévy models as defined in (1.1) provide a flexible and tractable modelling framework that recently has been used for a variety of modelling purposes, including modelling of turbulent flows ([10]), growth processes ([17]), Cox point processes ([16]), and brain imaging data ([18]). In [18], a model (1.1) with $M$ following a NIG distribution was suitable for modelling the neuroscience data under consideration. For such data it is typically of interest to detect for which $t \in B$ a given field obtains values that are significantly large.

To the best of our knowledge, the extremal behaviour of a NIG field or more generally a field (1.1) with convolution equivalent Lévy measure has not yet been studied in detail. For Gaussian random fields it is known that the distribution of the supremum of the field can be approximated by the expected Euler characteristic of an excursion set (see [4] and references therein). In [15] an exact asymptotic result is obtained for Gaussian random fields under the assumption of $\alpha(t)$-local stationarity. However, in [18] it is shown by simulations that using a model based on the NIG distribution gives results that are substantially different from those obtained by Gaussian models.

The supremum of a non-Gaussian field given by integrals with respect to an infinitely divisible random measure has already been studied, when the random measure has regularly varying tails. Results for the asymptotic distribution of the supremum are found in [25], and these results are refined in [2] and [3], where results are obtained on the asymptotic joint distribution of the number of critical points of the excursion sets. The arguments are - as in the present paper - based on finding the Lévy measure of a dense countable subset of the field. However, the remaining proofs rely heavily on the assumption of regularly varying tails and can therefore not be translated into the convolution equivalent framework.

Note that convolution equivalent distributions have heavier tails than Gaussian distributions and lighter tails than those of regularly varying distributions. The latter statement follows from the fact that convolution equivalent distributions have exponential tails while regularly varying distributions have power function tails.

For real-valued one dimensional infinitely divisible distributions it is shown in [11], [12] and [21] that if the Lévy measure has a convolution equivalent right tail, then the distribution has a right tail that is asymptotically equivalent. The proofs are based on a decomposition of the distribution into a compound Poisson part that is dominating in the tail and a part with a lighter tail. The arguments in the present paper applies a similar decomposition to the distribution of a dense countable subset of the field.

In [13], results for a moving average process on $\mathbb{R}$, obtained as an integral with respect to a Lévy process with convolution equivalent tail, are derived. But here the kernel function $f$ satisfies $f(t)=0$ for $t<0$ such that

$$
X_{t}=\int_{-\infty}^{t} f(t-s) M(\mathrm{~d} s) .
$$


The present paper is organised as follows. In Section 2, we give a short introduction to random fields defined as an integral of a kernel function with respect to a Lévy basis. Such a field $X$ can be decomposed into a sum $X^{1}+X^{2}+X^{3}$ of three independent fields, including a compound Poisson sum $X^{1}$ and a Gaussian part $X^{3}$. In Section 3, the tail asymptotics for $X^{1}$ is studied, while it is shown in Section 4 that the supremum of the fields $X^{2}$ and $X^{3}$ have lighter tails than the supremum of $X^{1}$. This makes it possible to derive the overall extremal behaviour of the supremum of $X$, which is also done in Section 4. The asymptotic behaviour of excursion sets is shortly discussed in Section 5. Proofs concerning the existence of continuous versions of the random fields considered are deferred to an Appendix.

\section{Preliminaries}

Consider an independently scattered random measure $M$ on $\mathbb{R}^{d}$. Then for a sequence of disjoint sets $\left(A_{n}\right)_{n \in \mathbb{N}} \subseteq \mathbb{R}^{d}$ in $\mathcal{B}\left(\mathbb{R}^{d}\right)$ the random variables $\left(M\left(A_{n}\right)\right)_{n \in \mathbb{N}}$ are independent and satisfy $M\left(\cup A_{n}\right)=\sum M\left(A_{n}\right)$. Assume furthermore that $M(A)$ is infinitely divisible for all $A \in \mathcal{B}\left(\mathbb{R}^{d}\right)$. Then $M$ is called a Lévy basis, see [10] and references therein.

For a random variable $X$ let $C(\lambda \ddagger X)$ denote its cumulant function $\log E\left(e^{i \lambda X}\right)$. We shall assume that the Lévy basis is stationary and isotropic such that for $A \in$ $\mathcal{B}\left(\mathbb{R}^{d}\right)$ the variable $M(A)$ has a Lévy-Khintchine representation given by

$C(\lambda \ddagger M(A))=i \lambda a m_{d}(A)+\frac{1}{2} \lambda^{2} \theta m_{d}(A)+\int_{A \times \mathbb{R}}\left(e^{i \lambda u}-1-i \lambda u 1_{[-1,1]}(u)\right) F(\mathrm{~d} s, \mathrm{~d} u)$,

where $m_{d}$ is the Lebesgue measure on $\left(\mathbb{R}^{d}, \mathcal{B}\left(\mathbb{R}^{d}\right)\right), a \in \mathbb{R}, \theta \geq 0$ and $F$ is a measure on $\mathcal{B}\left(\mathbb{R}^{d} \times \mathbb{R}\right)$ on the form

$$
F(A \times B)=\xi m_{d}(A) \rho(B) .
$$

We assume that $\rho$ has an exponentially varying right tail with parameter $\beta>0$

$$
\rho((x, \infty))=L(x) \mathrm{e}^{-\beta x},
$$

where $L$ satisfies $L(x) \sim x^{-\delta}$ with $\delta>1$. Here we use the convention that $f(x) \sim$ $g(x)$ if $f(x) / g(x) \rightarrow 1$ as $x \rightarrow \infty$. As noted in the introduction, this makes $\rho$ convolution equivalent. We furthermore assume

$$
\int z^{2} \rho(\mathrm{d} z)<\infty
$$

Note that the integrability along the right tail of $\rho$ follows from (2.3) and that in particular

$$
\int_{[-1,1]} z^{2} \rho(\mathrm{d} z)<\infty
$$

which is needed for $\rho$ to be a Lévy measure. Finally we assume that there exists another Lévy measure $\bar{\rho}$ on the form

$$
\bar{\rho}(\mathrm{d} x)=C|x|^{-r} \mathrm{~d} x
$$

with $r \in(2,3)$ and $C>0$, such that $\bar{\rho}-\rho$ is a non-negative measure. 
Example 2.1 (IG basis). Suppose that $M$ is inverse Gaussian,

$$
M(A) \sim I G\left(\eta m_{d}(A), \gamma\right),
$$

$\eta, \gamma>0$. Then $C(\lambda \ddagger M(A))$ has the representation (2.1) from above with

$$
\begin{aligned}
a & =\frac{\eta}{\gamma} \int_{0}^{1} x^{-1 / 2} e^{(-1 / 2) \gamma^{2} x} \mathrm{~d} x \\
\theta & =0 \\
\xi & =\frac{\eta}{\gamma^{2}} \sqrt{\frac{2}{\pi}} \\
\rho(\mathrm{d} x) & =\frac{\gamma^{2}}{2} 1_{\mathbb{R}_{+}}(x) x^{-3 / 2} e^{(-1 / 2) \gamma^{2} x} \mathrm{~d} x,
\end{aligned}
$$

see e.g., $[8,9,17]$. Thereby

$$
\rho((x, \infty))=\frac{\gamma^{2}}{2} \int_{x}^{\infty} y^{-3 / 2} e^{(-1 / 2) \gamma^{2} y} \mathrm{~d} y \sim x^{-3 / 2} e^{(-1 / 2) \gamma^{2} x}
$$

as $x \rightarrow \infty$.

Example 2.2 (NIG basis). Suppose that $M$ is normal inverse Gaussian,

$$
M(A) \sim N I G\left(\alpha, \beta, \mu m_{d}(A), \delta m_{d}(A)\right),
$$

$0 \leq|\beta|<\alpha, \mu \in \mathbb{R}$ and $0<\delta$. Then $C(\lambda \ddagger M(A))$ has the representation (2.1) from above with

$$
\begin{aligned}
a & =\mu+\frac{2 \delta \alpha}{\pi} \int_{0}^{1} \sinh (\beta x) K_{1}(\alpha x) \mathrm{d} x \\
\theta & =0 \\
\xi & =\frac{\delta}{\alpha-\beta} \sqrt{\frac{\alpha}{2 \pi}} \\
\rho(\mathrm{d} x) & =(\alpha-\beta) \sqrt{\frac{2 \alpha}{\pi}} \frac{1}{|x|} K_{1}(\alpha|x|) e^{\beta x} \mathrm{~d} x,
\end{aligned}
$$

where $K_{1}$ is the modified Bessel function of second kind and index 1. For further details concerning the Lévy measure of the NIG distribution, see [6] and [7]. Using the well-known asymptotic formula for $K_{1}$

we find

$$
K_{1}(s) \sim \sqrt{\frac{\pi}{2}} s^{-1 / 2} e^{-s} \quad \text { as } s \rightarrow \infty,
$$

$$
\sqrt{\frac{2 \alpha}{\pi}} \frac{1}{|x|} K_{1}(\alpha|x|) e^{\beta x} \sim|x|^{-3 / 2} e^{-\alpha|x|+\beta x}
$$

as $|x| \rightarrow \infty$ and thereby

$$
\begin{aligned}
\rho((x, \infty)) & \sim(\alpha-\beta) \int_{x}^{\infty}|y|^{-3 / 2} e^{-\alpha|y|+\beta y} \mathrm{~d} y \\
& \sim x^{-3 / 2} e^{-(\alpha-\beta) x}
\end{aligned}
$$

as $x \rightarrow \infty$. 
Now assume that $f:[0, \infty) \rightarrow[0, \infty)$ is an integration kernel satisfying

$$
f(0)=1, \quad f(x)<1 \text { for } x>0, \quad \int_{\mathbb{R}^{d}} f(|s|) \mathrm{d} s<\infty
$$

and

$$
f(x) \leq \frac{K_{1}}{(x+1)^{d}} \quad \text { for all } x \geq 0
$$

for a finite, positive constant $K_{1}$. Assume furthermore that $f$ is differentiable with $f^{\prime}$ satisfying

$$
\left|f^{\prime}(x)\right| \leq \frac{K_{2}}{(x+1)^{d}} \quad \text { for all } x \geq 0
$$

for a finite, positive constant $K_{2}$. Let $B$ be a compact subset of $\mathbb{R}^{d}$ with $m_{d}(B)>0$ and consider the family of random variables $\left(X_{t}\right)_{t \in B}$ defined by

$$
X_{t}=\int_{\mathbb{R}^{d}} f(|t-s|) M(\mathrm{~d} s) .
$$

The integrals defining each $X_{t}$ exists according to [24, Theorem 2.7], where the conditions (i)-(iii) can be easily verified under the given assumptions on $M$ and $f$. As explained in the Appendix, Theorem A.1, there furthermore exists a version of $\left(X_{t}\right)_{t \in B}$ with continuous sample paths. In the following, it will be useful to note that

$$
\int_{\mathbb{R}^{d}} \sup _{t \in B} f(|t-s|) \mathrm{d} s<\infty .
$$

Example 2.3 (Exponential kernel function). Suppose that

$$
f(x)=e^{-\sigma x},
$$

$\sigma>0$, then the assumptions (2.6)-(2.8) are satisfied.

Example 2.4 (Gaussian kernel function). Suppose that

$$
f(x)=e^{-\sigma x^{2}}
$$

$\sigma>0$, then the assumptions (2.6)-(2.8) are satisfied.

Example 2.5 (Matérn kernel function). Suppose that

$$
f(x)=\frac{1}{2^{\eta-1} \Gamma(\eta)}|\lambda x|^{\eta} K_{\eta}(\lambda|x|)
$$

where $K_{\eta}$ is the modified Bessel function of the second kind, index $\eta$, and $\lambda>0$. The use of this kernel function in Lévy based modelling and its relation to the so-called Matérn correlation structure of the field $\left(X_{t}\right)_{t \in B}$ have been discussed in [18]. For a further discussion of modelling, using a Matérn correlation structure, see [14]. Since for $\eta=\frac{1}{2}$

$$
K_{\frac{1}{2}}(x)=\sqrt{\frac{\pi}{2}} x^{-\frac{1}{2}} e^{-x}
$$


the Matérn kernel reduces to the exponential kernel for $\eta=\frac{1}{2}$. Below, we show for $\eta \geq \frac{1}{2}$ that the Matérn kernel satisfies the assumptions (2.6) $-(2.8)$. Since for $\eta>0$

$$
K_{\eta}(x) \sim 2^{\eta-1} \Gamma(\eta) x^{-\eta}
$$

as $x \downarrow 0$, we have $f(0)=1$. The integrability of $f$ is a result of the fact that for all $\eta>0$

$$
K_{\eta}(x) \sim \sqrt{\pi / 2} x^{-1 / 2} e^{-x}
$$

as $x \rightarrow \infty$. Using (2.10), it also follows that (2.6) is fulfilled. It remains to show that $f(x)<1$ for $x \neq 0$ and that (2.8) is fulfilled for $\eta \geq \frac{1}{2}$. Let us consider the function $f_{\eta}:[0, \infty) \rightarrow[0, \infty)$ defined by $f_{\eta}(x)=x^{\eta} K_{\eta}(x)$ for $\eta>0$. We apply the representation

$$
K_{\eta}(x)=\int_{0}^{\infty} \exp (-x \cosh t) \cosh (\eta t) \mathrm{d} t
$$

to obtain for $x>0$

$$
K_{\eta}^{\prime}(x)=-\int_{0}^{\infty} \exp (-x \cosh t) \cosh t \cosh (\eta t) \mathrm{d} t
$$

Using that $\cosh t \cosh (\eta t)=\frac{1}{2}(\cosh ((\eta+1) t)+\cosh ((\eta-1) t))$ and rearranging terms yields

$$
f_{\eta}^{\prime}(x)=\eta x^{\eta-1} K_{\eta}(x)+x^{\eta} K_{\eta}^{\prime}(x)=\eta x^{\eta-1} K_{\eta}(x)-\frac{x^{\eta}}{2}\left(K_{\eta+1}(x)+K_{\eta-1}(x)\right) .
$$

We can easily obtain the recurrence formula

$$
-x K_{\eta-1}(x)+x K_{\eta+1}(x)=2 \eta K_{\eta}(x),
$$

by using a similar recurrence formula for Bessel functions and the correspondence between Bessel functions and modified functions, see [5, Chapter 4]. Combining this with the expression for $f_{\eta}^{\prime}(x)$, we find

$$
f_{\eta}^{\prime}(x)=-x^{\eta} K_{\eta-1}(x) .
$$

From this we conclude, that $f_{\eta}^{\prime}$ is strictly negative on $(0, \infty)$. It follows that $f(x)<$ $f(0)$ for $x \neq 0$. Since furthermore for $\eta>0, x^{\eta} K_{\eta}(x) \rightarrow 2^{\eta-1} \Gamma(\eta)$ as $x \rightarrow \infty$ and $K_{\eta}=K_{-\eta}$, we deduce that $\lim _{x \rightarrow 0} f_{\eta}^{\prime}(x)=0$ for $\eta>\frac{1}{2}$ and $\lim _{x \rightarrow 0} f_{\eta}^{\prime}(x)=-\infty$ for $0<\eta<\frac{1}{2}$. For $\eta=\frac{1}{2}$ we have that $f_{\frac{1}{2}}(x)=\sqrt{\frac{\pi}{2}} e^{-x}$, so $f_{\frac{1}{2}}^{\prime}(0)=-\sqrt{\frac{\pi}{2}}$. Combining this with (2.10) we obtain the desired inequality (2.8) for $f^{\prime}$, when $\eta \geq 1 / 2$.

For the study of the extremal behaviour of $\left(X_{t}\right)_{t \in B}$, we will use that the cumulant function of $X_{t}=\int_{\mathbb{R}^{d}} f(|t-s|) M(\mathrm{~d} s)$ takes the following form

$$
\begin{aligned}
C\left(\lambda \ddagger X_{t}\right)= & i \lambda a \int_{\mathbb{R}^{d}} f(|t-s|) \mathrm{d} s+\frac{1}{2} \lambda^{2} \theta \int_{\mathbb{R}^{d}} f(|t-s|)^{2} \mathrm{~d} s \\
& +\xi \int_{\mathbb{R}^{d}} \int_{\mathbb{R}}\left(e^{i f(|t-s|) \lambda u}-1-i f(|t-s|) \lambda u 1_{[-1,1]}(u)\right) \rho(\mathrm{d} u) \mathrm{d} s,
\end{aligned}
$$


cf. e.g., [24, Theorem 2.7]. A similar formula holds for finite linear combinations of the $X_{t}$ s. Here, $f(|t-s|)$ is substituted by $\sum_{t} \beta_{t} f(|t-s|)$. It follows that all finite dimensional distributions of $\left(X_{t}\right)_{t \in B}$ are infinitely divisible. As a consequence, any countable field $\left(X_{t}\right)_{t \in T}$ is itself infinitely divisible, see [20] for existence and uniqueness of the infinite divisibility of the entire field. It follows from direct manipulations and it is also noted in e.g., [25] that the Lévy measure of $\left(X_{t}\right)_{t \in T}$ is the measure $\nu$ on $\left(\mathbb{R}^{T}, \mathcal{B}\left(\mathbb{R}^{T}\right)\right)$ defined by $\nu=F \circ V^{-1}$, where $V: \mathbb{R}^{d} \times \mathbb{R} \rightarrow \mathbb{R}^{T}$ is given by

$$
V(s, z)=(z f(|t-s|))_{t \in T} .
$$

We will from now on assume that $T=B \cap \mathbb{Q}^{d}$, where $\mathbb{Q}^{d}$ are the rational numbers in $\mathbb{R}^{d}$. For $\beta \in \mathbb{R}^{T}$ with $\beta_{t}=0$ for all but finitely many $t \in T$ we find

$$
\begin{aligned}
\log E\left(\exp \left(i \sum_{t} \beta_{t} X_{t}\right)\right)= & i \sum_{t} \beta_{t} a_{t}+\frac{1}{2} \theta \int_{\mathbb{R}^{d}}\left(\sum_{t} \beta_{t} f(|t-s|)\right)^{2} \mathrm{~d} s \\
& +\int_{\mathbb{R}^{T}}\left(\exp \left(i \sum_{t} \beta_{t} x_{t}\right)-1-i \sum_{t} \beta_{t} x_{t} 1_{[-1,1]^{T}}(x)\right) \nu(\mathrm{d} x)
\end{aligned}
$$

for an appropriate choice of $\left(a_{t}\right)_{t \in T} \in \mathbb{R}^{T}$. It is furthermore seen that $\left(a_{t}\right)_{t \in T}$ is bounded. Because of the infinite divisibility of $\left(X_{t}\right)_{t \in T}$, we have the following decomposition, see e.g., [25],

$$
X_{t}=X_{t}^{1}+X_{t}^{2}+X_{t}^{3}
$$

where the fields $\left(X_{t}^{1}\right)_{t \in T},\left(X_{t}^{2}\right)_{t \in T}$ and $\left(X_{t}^{3}\right)_{t \in T}$ are independent. The first field $\left(X_{t}^{1}\right)_{t \in T}$ is a compound Poisson sum

$$
X_{t}^{1}=\sum_{n=0}^{N} U_{t}^{n},
$$

where $N$ is Poisson distributed with parameter $\nu(A)$ and

$$
A=\left\{x \in \mathbb{R}^{T}: \sup _{t \in T} x_{t} \geq 1\right\} .
$$

In the Appendix, it is shown that $\nu(A)<\infty$, see Lemma A.3. The fields $\left(U_{t}^{n}\right)_{t \in T}$ are independent and identically distributed with common distribution $\nu_{1}=\nu_{A} / \nu(A)$, where $\nu_{A}$ is the measure on $\left(\mathbb{R}^{T}, \mathcal{B}\left(\mathbb{R}^{T}\right)\right)$ obtained by restricting $\nu$ to $A$. The distribution of $\left(X_{t}^{1}\right)_{t \in T}$ is also determined by the following cumulant functions

$$
\log \left(\exp \left(i \sum_{t} \beta_{t} X_{t}^{1}\right)\right)=\int_{\mathbb{R}^{T}}\left(\exp \left(i \sum_{t} \beta_{t} x_{t}\right)-1\right) \nu_{A}(\mathrm{~d} x)
$$

where $\beta \in \mathbb{R}^{T}$ satisfies $\beta_{t}=0$ for all $t \notin T_{0}$. Furthermore $\left(X_{t}^{2}\right)_{t \in T}$ is infinitely divisible with a Lévy measure $\nu_{A^{c}}$, the restriction of $\nu$ to $A^{c}$, and cumulant function given by

$$
\begin{aligned}
\log E & \left(\exp \left(i \sum_{t} \beta_{t} X_{2}^{1}\right)\right) \\
& =i \sum_{t} \beta_{t} a_{t}+\int_{\mathbb{R}^{T}}\left(\exp \left(i \sum_{t} \beta_{t} x_{t}\right)-1-i \sum_{t} \beta_{t} x_{t} 1_{[-1,1]^{T}}(x)\right) \nu_{A}^{c}(\mathrm{~d} x),
\end{aligned}
$$


where $\beta \in \mathbb{R}^{T}$ with $\beta_{t}=0$ for all but finitely many $t \in T$. Finally $\left(X_{t}^{3}\right)_{t \in T}$ is defined by

$$
X_{t}^{3}=\int_{\mathbb{R}^{d}} f(|t-s|) M^{3}(\mathrm{~d} s),
$$

where $M^{3}$ is a Gaussian Lévy basis satisfying $C\left(\lambda \ddagger M^{3}(A)\right)=i \lambda^{2} \theta m_{d}(A)$.

It will be crucial for the arguments in the following sections that each of the fields $X^{1}, X^{2}$, and $X^{3}$ can be extended to continuous fields indexed by $B$. Note that each of the fields $\left(U_{t}^{n}\right)_{t \in T}$ almost surely has the form $z f(|t-s|)$. Hence there almost surely exists a continuous extension $\left(U_{t}^{n}\right)_{t \in B}$. Since $X^{1}$ is a finite sum of such fields it has a continuous extension to $B$ as well. As already stated, the field $\left(X_{t}\right)_{t \in B}$ has continuous sample paths, see also Theorem A.1. Furthermore, $\left(X_{t}^{3}\right)_{t \in B}$ has a continuous version under the given assumptions on $f$, see the Appendix, Theorem A.2. Thereby also $X^{2}$ has continuous sample paths.

\section{Tail asymptotics for compound Poisson sum of Lévy fields}

In this section, we will determine the extremal behaviour of $P\left(X_{t}^{1}>x-y_{t}\right.$ for some $\left.t\right)$ for increasing values of $x$ and $\left(y_{t}\right)_{t \in B}$ a continuous field. The main result, formulated in Theorem 3.5 below, will be used in the next section to study the extremal behaviour of $P\left(\sup _{t \in B} X_{t}>x\right)$, using the fact that $X=X^{1}+X^{2}+X^{3}$ and conditioning on $X^{2}$ and $X^{3}$.

It is convenient to introduce a notation that can be seen as a refinement of the event $\left\{\sup _{t \in T} X_{t}>x\right\}$. If $\left(x_{t}\right)_{t \in T}$ is a field in $\mathbb{R}^{T}$, we define $\Gamma\left(\left(x_{t}\right)_{t \in T}\right)$ to be the following subset in $\mathcal{B}\left(\mathbb{R}^{T}\right)$

$$
\Gamma\left(\left(x_{t}\right)_{t \in T}\right)=\left\{\left(y_{t}\right)_{t \in T}: y_{t}>x_{t} \text { for some } t \in T\right\} .
$$

If $x_{t}=x$ for all $t \in T$ we shall use the notation $\Gamma(x)$. Note that $\left\{\sup _{t \in T} X_{t}>x\right\}=$ $\{X \in \Gamma(x)\}$.

The first step will be determining the behaviour of $P\left(U \in \Gamma\left(\left(x-y_{t}\right)_{t \in T}\right)\right)$, when $U$ is a field with distribution $\nu_{1}$.

Theorem 3.1. Let $\left(y_{t}\right)_{t \in B}$ be continuous and bounded on B. Then

$$
\frac{\nu_{1}\left(\Gamma\left(\left(x-y_{t}\right)_{t \in T}\right)\right)}{L(x) \exp (-\beta x)} \rightarrow \frac{\xi}{\nu(A)} \int_{B} \exp \left(\beta y_{s}\right) \mathrm{d} s \quad \text { as } x \rightarrow \infty .
$$

Furthermore,

$$
\frac{\nu_{1}(\Gamma(x))}{L(x) \exp (-\beta x)} \rightarrow \frac{\xi}{\nu(A)} m_{d}(B) \quad \text { as } x \rightarrow \infty
$$

and

$$
\frac{\nu_{1}\left(\Gamma\left(\left(x-y_{t}\right)_{t \in T}\right)\right)}{\nu_{1}(\Gamma(x))} \rightarrow \frac{\int_{B} \exp \left(\beta y_{s}\right) \mathrm{d} s}{m_{d}(B)} \quad \text { as } x \rightarrow \infty
$$


Proof. The results (3.2) and (3.3) are direct consequences of (3.1), so we focus on the proof of (3.1). We can assume that $\left(y_{t}\right)_{t \in B}$ is non-negative: Simply write $x=x^{\prime}-x_{0}$ for a suitable $x_{0}$ such that $\left(x_{0}+y_{t}\right)_{t \in B}$ is non-negative, and find the limit of

$$
\frac{\nu_{1}\left(\Gamma\left(\left(x^{\prime}-\left(x_{0}+y_{t}\right)\right)_{t \in T}\right)\right)}{L\left(x^{\prime}\right) \exp \left(-\beta x^{\prime}\right)}
$$

as $x^{\prime} \rightarrow \infty$. We find

$$
\begin{aligned}
& \nu_{1}\left(\Gamma\left(\left(x-y_{t}\right)_{t \in T}\right)\right) \\
&=\frac{1}{\nu(A)} F \circ V^{-1}\left(\Gamma\left(\left(x-y_{t}\right)_{t \in T}\right)\right) \\
&=\frac{1}{\nu(A)} F\left(\left\{(s, z) \in \mathbb{R}^{d} \times \mathbb{R}: \exists t \in T: z f(|t-s|)>x-y_{t}\right\}\right) \\
&=\frac{1}{\nu(A)} F\left(\left\{(s, z) \in \mathbb{R}^{d} \times \mathbb{R}: z>\inf _{t \in T} \frac{x-y_{t}}{f(|t-s|)}\right\}\right) \\
&=\frac{\xi}{\nu(A)} \int_{\mathbb{R}^{d}} L\left(\inf _{t \in T} \frac{x-y_{t}}{f(|t-s|)}\right) \exp \left(-\beta \inf _{t \in T} \frac{x-y_{t}}{f(|t-s|)}\right) \mathrm{d} s \\
&=\frac{\xi}{\nu(A)} \int_{B} L\left(\inf _{t \in T} \frac{x-y_{t}}{f(|t-s|)}\right) \exp \left(-\beta \inf _{t \in T} \frac{x-y_{t}}{f(|t-s|)}\right) \mathrm{d} s \\
&+\frac{\xi}{\nu(A)} \int_{\mathbb{R}^{d} \backslash B} L\left(\inf _{t \in T} \frac{x-y_{t}}{f(|t-s|)}\right) \exp \left(-\beta \inf _{t \in T} \frac{x-y_{t}}{f(|t-s|)}\right) \mathrm{d} s .
\end{aligned}
$$

First, we show that the second term in (3.4) is $o(L(x) \exp (-\beta x))$. Since $L(x) \sim x^{-\delta}$, $\delta>1$, there exists a constant $C$ such that $L(x) \leq C x^{-\delta}$ for all $x>0$. With the notation $y^{*}=\sup _{s} y_{s}$ the second term is

$$
\begin{aligned}
& \leq C \frac{\xi}{\nu(A)} \int_{\mathbb{R}^{d} \backslash B}\left(\inf _{t \in T} \frac{x-y^{*}}{f(|t-s|)}\right)^{-\delta} \exp \left(-\beta \inf _{t \in T} \frac{x-y^{*}}{f(|t-s|)}\right) \mathrm{d} s \\
& =C \frac{\xi}{\nu(A)} \int_{\mathbb{R}^{d} \backslash B}\left(\frac{x-y^{*}}{\sup _{t \in T} f(|t-s|)}\right)^{-\delta} \exp \left(-\beta \frac{x-y^{*}}{\sup _{t \in T} f(|t-s|)}\right) \mathrm{d} s .
\end{aligned}
$$

The integrand in (3.5) is clearly $o(L(x) \exp (-\beta x))$, since $\sup _{t \in T} f(|t-s|)<1$ for all $s \in \mathbb{R}^{d} \backslash B$. If we denote the integrand of $(3.5)$ by $h(s ; x)$, it follows by the dominated convergence theorem that $(3.5)$ is $o(L(x) \exp (-\beta x))$ if we can find an integrable function $g$ such that

$$
\frac{h(s ; x)}{x^{-\delta} \exp (-\beta x)} \leq g(s), \quad s \in \mathbb{R}^{d} .
$$

Using the notation $f_{0}(s)=\sup _{t \in T} f(|t-s|)$, we find for $x>2 y^{*}$

$$
\begin{aligned}
\frac{h(s ; x)}{x^{-\delta} \exp (-\beta x)} & =\left(\frac{x-y^{*}}{x}\right)^{-\delta} \exp \left(\beta y^{*}\right) f_{0}(s)^{\delta} \exp \left(-\beta\left(\frac{1}{f_{0}(s)}-1\right)\left(x-y^{*}\right)\right) \\
& \leq 2^{\delta} \exp \left(\beta y^{*}\right) \exp \left(-\beta\left(\frac{1}{f_{0}(s)}-1\right) y^{*}\right) .
\end{aligned}
$$


Now, choose $r>0$ such that $B \subseteq C_{r}(0)$, where $C_{r}(0)$ is the ball with radius $r$ and centre $0 \in \mathbb{R}^{d}$. Then, using (2.7), we get for $s \notin C_{r}(0)$

$$
f_{0}(s) \leq \sup _{t \in C_{r}(0)} f(|t-s|) \leq \sup _{t \in C_{r}(0)} \frac{1}{(|t-s|+1)^{d}}=\frac{1}{(|s|-r+1)^{d}}
$$

It follows that the function (3.6) is integrable.

The theorem now follows from applying dominated convergence to the first term of (3.4). Since for $s \in B$

$$
\inf _{t \in T} \frac{x-y_{t}}{f(|t-s|)}-\left(x-y_{s}\right) \rightarrow 0 \quad \text { as } x \rightarrow \infty
$$

we have

$$
L\left(\inf _{t \in T} \frac{x-y_{t}}{f(|t-s|)}\right) \exp \left(-\beta \inf _{t \in T} \frac{x-y_{t}}{f(|t-s|)}\right) \sim L\left(x-y_{s}\right) \exp \left(-\beta\left(x-y_{s}\right)\right),
$$

SO

$$
\frac{L\left(\inf _{t \in T} \frac{x-y_{t}}{f(|t-s|)}\right) \exp \left(-\beta \inf _{t \in T} \frac{x-y_{t}}{f(|t-s|)}\right)}{L(x) \exp (-\beta x)} \rightarrow e^{\beta y_{s}},
$$

and for $x>2 y^{*}$

$$
\begin{aligned}
& \left|\frac{L\left(\inf _{t \in T} \frac{x-y_{t}}{f(|t-s|)}\right) \exp \left(-\beta \inf _{t \in T} \frac{x-y_{t}}{f(|t-s|)}\right)}{L(x) \exp (-\beta x)}-e^{\beta y_{s}}\right| \\
& \quad \leq \frac{C}{c} \frac{\left(\inf _{t \in T} \frac{x-y^{*}}{f(|t-s|)}\right)^{-\delta} \exp \left(-\beta \inf _{t \in T} \frac{x-y^{*}}{f(|t-s|)}\right)}{x^{-\delta} \exp (-\beta x)}+e^{\beta y_{s}} \\
& \quad \leq \frac{C}{c}\left(\frac{x}{x-y^{*}}\right)^{\delta} e^{\beta y^{*}}+e^{\beta y_{s}} \\
& \quad \leq \frac{C}{c} 2^{\delta} e^{\beta y^{*}}+e^{\beta y_{s}}
\end{aligned}
$$

which is integrable over $B$. Above $c$ is chosen such that $c x^{-\delta} \leq L(x)$ for $x>2 y^{*}$.

Below, we extend the result of Theorem 3.1 to the case $P\left(U_{1}+\cdots+U_{n} \in\right.$ $\left.\Gamma\left(\left(x-y_{t}\right)_{t \in T}\right)\right)$, where $U_{i}, i=1, \ldots, n$, are independent with common distribution $\nu_{1}$. For this purpose, we need the lemma below.

Lemma 3.2. We have

$$
\int e^{\beta \sup _{t} z_{t}} \nu_{1}(\mathrm{~d} z)<\infty
$$

Proof. Let $\left(Z_{t}\right)_{t \in T}$ be distributed according to $\nu_{1}$. Then $Z^{*}=\sup _{t} Z_{t}$ satisfies

$$
P\left(Z^{*}>x\right) \sim C_{1} x^{-\delta} e^{-\beta x} \quad \text { as } x \rightarrow \infty,
$$


see Theorem 3.1. It therefore suffices to show that $E\left(e^{\beta Z^{*}}\right)$ is finite for a random variable $Z^{*}$ satisfying (3.7). For a suitable constant $C^{\prime}>C$ we have that $P(Z>x) \leq$ $C^{\prime} x^{-\delta} e^{-\beta x}$ for all $x>0$. Then,

$$
\begin{aligned}
E\left(e^{\beta Z^{*}}\right) & =\int_{0}^{\infty} P\left(e^{\beta Z^{*}}>x\right) \mathrm{d} x \\
& =\int_{0}^{\infty} P\left(Z^{*}>\frac{\log x}{\beta}\right) \mathrm{d} x \leq 1+C^{\prime} \beta^{\delta} \int_{1}^{\infty} \frac{(\log x)^{-\delta}}{x} \mathrm{~d} x,
\end{aligned}
$$

which is finite.

If $\left(U_{t}\right)_{t \in T}$ and $\left(V_{t}\right)_{t \in T}$ are independent random fields with distributions $\nu$ and $\mu$ on $\left(\mathbb{R}^{T}, \mathcal{B}\left(\mathbb{R}^{T}\right)\right)$, then we will use the notation $\nu * \mu$ for the distribution of $\left(U_{t}+V_{t}\right)_{t \in T}$. Similarly, we write $\nu^{* n}$ for the $n$-fold convolution of $\nu$. We have

Theorem 3.3. For all $n \geq \mathbb{N}$ and $\left(y_{t}\right)_{t \in T}$ bounded and continuous, it holds that

$$
\frac{\nu_{1}^{* n}\left(\Gamma\left(\left(x-y_{t}\right)_{t \in T}\right)\right.}{\nu_{1}(\Gamma(x))} \rightarrow \frac{n}{m_{d}(B)}\left(\int_{B} e^{\beta y_{s}} \int e^{\beta z_{s}} \nu_{1}^{*(n-1)}(\mathrm{d} z) \mathrm{d} s\right)
$$

as $x \rightarrow \infty$.

Proof. In the proof we shall use the notation

$$
K=\int e^{\beta \sup _{t} z_{t}} \nu_{1}(\mathrm{~d} z)
$$

The result is shown by induction over $n$. For $n=1$, the result is shown in Theorem 3.1. Assume now that the theorem is correct for some $n \in \mathbb{N}$. Let $\left(U_{t}\right)_{t \in T}$ and $\left(V_{t}\right)_{t \in T}$ be independent and with distribution $\nu_{1}$ and $\nu_{1}^{* n}$, respectively. Then we have

$$
\begin{aligned}
\left(\nu_{1}^{* n} * \nu_{1}\right) & \left(\Gamma\left(\left(x-y_{t}\right)_{t \in T}\right)\right) \\
= & P\left(\exists t: U_{t}+V_{t}>x-y_{t}\right) \\
= & P\left(\exists t: U_{t}>\left(x-y_{t}\right) / 2, \exists t: V_{t}>\left(x-y_{t}\right) / 2, \exists t: U_{t}+V_{t}>x-y_{t}\right) \\
& +P\left(\forall t: U_{t}<\left(x-y_{t}\right) / 2, \exists t: U_{t}+V_{t}>x-y_{t}\right) \\
& +P\left(\forall t: V_{t}<\left(x-y_{t}\right) / 2, \exists t: U_{t}+V_{t}>x-y_{t}\right) .
\end{aligned}
$$

The first term is bounded from above by

$$
\begin{aligned}
P(\exists t & \left.: U_{t}>\left(x-y_{t}\right) / 2, \exists t: V_{t}>\left(x-y_{t}\right) / 2\right) \\
& =\nu_{1}\left(\Gamma\left(\left(\left(x-y_{t}\right) / 2\right)_{t \in T}\right)\right) \nu_{1}^{* n}\left(\Gamma\left(\left(\left(x-y_{t}\right) / 2\right)_{t \in T}\right)\right) .
\end{aligned}
$$

This is $o\left(\nu_{A}(\Gamma(x))\right)$ according to Theorem 3.1 and the induction assumption.

For the evaluation of the second term in (3.8), we can assume that all the fields $z=\left(z_{t}\right)_{t \in T}$ have continuous extensions to $B$, since the distribution $\nu_{1}$ is concentrated on a set of fields with this property. The second term in (3.8) can be rewritten as 
follows

$$
\begin{aligned}
\int_{\left\{z: z_{t}<\left(x-y_{t}\right) / 2 \forall t\right\}} \nu_{1}^{* n}\left(\Gamma\left(\left(x-y_{t}-z_{t}\right)_{t \in T}\right)\right) \nu_{1}(\mathrm{~d} z) \\
\sim \int_{\left\{z: z_{t}<\left(x-y_{t}\right) / 2 \forall t\right\}} \frac{n}{m_{d}(B)} \nu_{1}(\Gamma(x))\left(\int_{B} e^{\beta\left(y_{s}+z_{s}\right)} \int e^{\beta u_{s}} \nu_{1}^{*(n-1)}(\mathrm{d} u) \mathrm{d} s\right) \nu_{1}(\mathrm{~d} z) \\
\sim \frac{n}{m_{d}(B)} \nu_{1}(\Gamma(x)) \int\left(\int_{B} e^{\beta\left(y_{s}+z_{s}\right)} \int e^{\beta u_{s}} \nu_{1}^{*(n-1))}(\mathrm{d} u) \mathrm{d} s\right) \nu_{1}(\mathrm{~d} z) \\
=\frac{n}{m_{d}(B)} \nu_{1}(\Gamma(x))\left(\int_{B} e^{\beta y_{s}} \int e^{\beta z_{s}} \nu_{1}^{* n}(\mathrm{~d} z) \mathrm{d} s\right) .
\end{aligned}
$$

The second asymptotic equivalence above is a simple result of the finiteness of $\nu_{1}$. The first asymptotic equivalence follows if $\int_{C_{x, y}} h(x, y, z) \nu_{1}(\mathrm{~d} z)$ has limit 0 , where $C_{x, y}=\left\{z: z_{t}<\left(x-y_{t}\right) / 2 \forall t\right\}$ and $h(x, y, z)$ is given by

$$
\frac{\nu_{1}^{* n}\left(\Gamma\left(\left(x-y_{t}-z_{t}\right)_{t \in T}\right)\right)-\frac{n}{m_{d}(B)} \nu_{1}(\Gamma(x))\left(\int_{B} e^{\beta\left(y_{s}+z_{s}\right)} \int e^{\beta u_{s}} \nu_{1}^{*(n-1)}(\mathrm{d} u) \mathrm{d} s\right)}{\nu_{1}(\Gamma(x))} .
$$

From the induction assumption the integrand has limit 0. There exists constants $0<c<C$ and $0<c^{\prime}<C^{\prime}$ such that for all $x \geq 1$

$$
\begin{gathered}
c x^{-\delta} e^{-\beta x} \leq \nu_{1}(\Gamma(x)) \leq C x^{-\delta} e^{-\beta x}, \\
c^{\prime} x^{-\delta} e^{-\beta x} \leq \nu_{1}^{* n}(\Gamma(x)) \leq C^{\prime} x^{-\delta} e^{-\beta x} .
\end{gathered}
$$

Hence $1_{C_{x, y}}(z) h(x, y, z)$ is numerically bounded from above by

$$
\begin{aligned}
& 1_{C_{x, y}}(z) \frac{\nu_{1}^{* n}\left(\Gamma\left(x-\sup _{t} y_{t}-\sup _{t} z_{t}\right)\right)+\frac{n K^{n-1}}{m_{d}(B)} \nu_{1}(\Gamma(x)) \int_{B} \exp \left(\beta\left(\sup _{t} y_{t}+\sup _{t} z_{t}\right)\right) \mathrm{d} s}{\nu_{1}(\Gamma(x))} \\
& \leq 1_{C_{x, y}}(z)\left[\frac{C^{\prime}}{c} \frac{\left(\left(x-3 \sup _{t}\left|y_{t}\right|\right) / 2\right)^{-\delta}}{x^{-\delta}}+n K^{n-1}\right] \exp \left(\beta \sup _{t} y_{t}\right) \exp \left(\beta \sup _{t} z_{t}\right),
\end{aligned}
$$

where we have used that $\sup _{t} z_{t} \leq\left(x+\sup _{t}\left|y_{t}\right|\right) / 2$ on $C_{x, y}$. Since $\frac{\left(\left(x-3 \sup _{t}\left|y_{t}\right|\right) / 2\right)^{-\delta}}{x^{-\delta}}$ is bounded as $x \rightarrow \infty$ and the second factor is integrable with respect to $\nu_{1}(\mathrm{~d} z)$, according to Lemma 3.2, then dominated convergence yields that $\int_{C_{x, y}} h(x, y, z) \nu_{1}(\mathrm{~d} z)$ has limit 0 .

The third term in (3.8) equals

$$
\begin{aligned}
\int_{\left\{z: z_{t}<\left(x-y_{t}\right) / 2 \forall t\right\}} \nu_{1}\left(\Gamma\left(\left(x-y_{t}-z_{t}\right)_{t \in T}\right)\right) \nu_{1}^{* n}(\mathrm{~d} z) \\
\quad \sim \int_{\left\{z: z_{t}<\left(x-y_{t}\right) / 2 \forall t\right\}} \frac{\nu_{1}(\Gamma(x))}{m_{d}(B)}\left(\int_{B} e^{\beta\left(y_{s}+z_{s}\right)} \mathrm{d} s\right) \nu_{1}^{* n}(\mathrm{~d} z) \\
\sim \frac{1}{m_{d}(B)} \nu_{1}(\Gamma(x))\left(\int_{B} e^{\beta y_{s}} \int e^{\beta z_{s}} \nu_{1}^{* n}(\mathrm{~d} z) \mathrm{d} s\right)
\end{aligned}
$$

where the asymptotic equivalences are obtained in the same way as to above. 
We are now ready to prove the main result of this section concerning the extremal behaviour of $P\left(X \in \Gamma\left(\left(x-y_{t}\right)_{t \in T}\right)\right)$ for large $x$. For a dominated convergence argument, we need the Lemma below.

Lemma 3.4. There exists a constant $K$ such that for all $n \in \mathbb{N}$ and all $x \geq 1$

$$
\nu_{1}^{* n}(\Gamma(x)) \leq K^{n} \nu_{1}(\Gamma(x)) .
$$

Proof. Note that there exists constants $0<c<C$ such that $c x^{-\delta} e^{-\beta x} \leq \nu_{1}(\Gamma(x))$ for all $x \geq 1$ and $\nu_{1}(\Gamma(x)) \leq C x^{-\delta} e^{-\beta x}$ for all $x>0$. Define

$$
K=\frac{C^{2} 4^{\delta}}{c}+2 \frac{C 2^{\delta}}{c}\left(\int \exp \left(\beta \sup _{t} y_{t}\right) \nu_{1}(\mathrm{~d} y)\right)
$$

and note that $K \geq 1$ so the result is true for $n=1$. Now the result is obtained by induction following the lines of the proof of Theorem 3.3. Assuming the result for $n \in \mathbb{N}$ we have

$$
\begin{aligned}
\nu_{1}^{*(n+1)}(\Gamma(x)) \leq & \nu_{1}^{* n}(\Gamma(x / 2)) \nu_{1}(\Gamma(x / 2)) \\
& +\int_{\left\{y: \sup y_{t} \leq x / 2\right\}} \nu_{1}^{* n}\left(\Gamma\left(\left(x-y_{t}\right)_{t \in T}\right)\right) \nu_{1}(\mathrm{~d} y) \\
& +\int_{\left\{y: \sup y_{t} \leq x / 2\right\}} \nu_{1}\left(\Gamma\left(\left(x-y_{t}\right)_{t \in T}\right)\right) \nu_{1}^{* n}(\mathrm{~d} y) .
\end{aligned}
$$

First, we observe that for $x \geq 1$

$$
\begin{gathered}
\nu_{1}^{* n}(\Gamma(x / 2)) \nu_{1}(\Gamma(x / 2)) \leq K^{n} \nu_{1}(\Gamma(x / 2))^{2} \leq K^{n} C^{2} 4^{\delta} x^{-2 \delta} e^{-\beta x} \\
\leq K^{n} C^{2} 4^{\delta} x^{-\delta} e^{-\beta x} \leq K^{n} \frac{C^{2} 4^{\delta}}{c} \nu_{1}(\Gamma(x))
\end{gathered}
$$

and secondly, we have

$$
\begin{aligned}
\int_{\left\{y: \sup y_{t} \leq x / 2\right\}} \nu_{1}^{* n}\left(\Gamma\left(\left(x-y_{t}\right)_{t \in T}\right)\right) \nu_{1}(\mathrm{~d} y) \\
\quad \leq \int_{\left\{y: \sup y_{t} \leq x / 2\right\}} \nu_{1}^{* n}\left(\Gamma\left(x-\sup _{t} y_{t}\right)\right) \nu_{1}(\mathrm{~d} y) \\
\quad \leq K^{n} C \int_{\left\{y: \sup y_{t} \leq x / 2\right\}}\left(x-\sup _{t} y_{t}\right)^{-\delta} \exp \left(-\beta\left(x-\sup _{t} y_{t}\right)\right) \nu_{1}(\mathrm{~d} y) \\
\quad \leq K^{n} C x^{-\delta} e^{-\beta x} \int_{\left\{y: \sup y_{t} \leq x / 2\right\}}\left(\frac{x / 2}{x}\right)^{-\delta} \exp \left(\beta \sup _{t} y_{t}\right) \nu_{1}(\mathrm{~d} y) \\
\leq K^{n} C 2^{\delta}\left(\int \exp \left(\beta \sup _{t} y_{t}\right) \nu_{1}(\mathrm{~d} y)\right) x^{-\delta} e^{-\beta x} \nu_{t} \nu_{1}(\Gamma(x)) .
\end{aligned}
$$


Finally, using the same type of arguments, we find

$$
\begin{aligned}
& \int_{\left\{y: \sup y_{t} \leq x / 2\right\}} \nu_{1}\left(\Gamma\left(\left(x-y_{t}\right)_{t \in T}\right)\right) \nu_{1}^{* n}(\mathrm{~d} y) \\
& \leq C 2^{\delta}\left(\int \exp \left(\beta \sup _{t} y_{t}\right) \nu_{1}^{* n}(\mathrm{~d} y)\right) x^{-\delta} e^{-\beta x} \\
& \leq \frac{C 2^{\delta}}{c}\left(\int \exp \left(\beta \sup _{t} y_{t}\right) \nu_{1}(\mathrm{~d} y)\right)^{n} \nu_{1}(\Gamma(x)) \leq \frac{C 2^{\delta}}{c} K^{n} \nu_{1}(\Gamma(x)) .
\end{aligned}
$$

Combining the inequalities gives the result of the lemma for $n+1$.

Recall that $\left(U_{t}^{n}\right)_{t \in T}$ are independent and identically distributed fields with common distribution $\nu_{1}$, and that $N$ is independent of the $U^{n}$ fields and Poisson distributed with parameter $\nu(A)$. We have defined $X^{1}$ by

$$
X_{t}^{1}=\sum_{n=0}^{N} U_{t}^{n}
$$

Theorem 3.5. We have $E \exp \left(\beta \sup _{t} X_{t}^{1}\right)<\infty$ and for a continuous field, $\left(y_{t}\right)_{t \in B}$

$$
\lim _{x \rightarrow \infty} \frac{P\left(X^{1} \in \Gamma\left(\left(x-y_{t}\right)_{t \in T}\right)\right.}{\nu(\Gamma(x))}=\frac{\int_{B} e^{\beta y_{s}} E\left(e^{\beta X_{s}^{1}}\right) \mathrm{d} s}{m_{d}(B)} .
$$

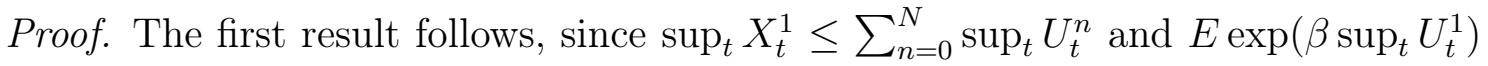
is finite.

For the proof of the limit result, we use that

$$
P\left(X^{1} \in \Gamma\left(\left(x-y_{t}\right)_{t \in T}\right)\right)=e^{-\nu(A)} \sum_{n=1}^{\infty} \frac{\nu(A)^{n}}{n !} \nu_{1}^{* n}\left(\Gamma\left(\left(x-y_{t}\right)_{t \in T}\right)\right) .
$$

Utilising Lemma 3.4, we find

$$
\begin{aligned}
& \sum_{n=1}^{\infty} \frac{\nu(A)^{n}}{n !} \frac{\nu_{1}^{* n}\left(\Gamma\left(\left(x-y_{t}\right)_{t \in T}\right)\right)}{\nu_{1}\left(\Gamma\left(x-\sup _{t} y_{t}\right)\right)} \leq \sum_{n=1}^{\infty} \frac{\nu(A)^{n}}{n !} \frac{\nu_{1}^{* n}\left(\Gamma\left(x-\sup _{t} y_{t}\right)\right)}{\nu_{1}\left(\Gamma\left(x-\sup _{t} y_{t}\right)\right)} \\
& \leq \sum_{n=1}^{\infty} \frac{K^{n} \nu(A)^{n}}{n !} \frac{\nu_{1}\left(\Gamma\left(x-\sup _{t} y_{t}\right)\right)}{\nu_{1}\left(\Gamma\left(x-\sup _{t} y_{t}\right)\right)}=\sum_{n=1}^{\infty} \frac{K^{n} \nu(A)^{n}}{n !}<\infty
\end{aligned}
$$

and, furthermore, we obtain from Theorem 3.3 that

$$
\lim _{x \rightarrow \infty} \frac{\nu_{1}^{* n}\left(\Gamma\left(\left(x-y_{t}\right)_{t \in T}\right)\right)}{\nu_{1}\left(\Gamma\left(x-\sup _{t} y_{t}\right)\right)}=\frac{n}{e^{\beta \sup _{t} y_{t}} m_{d}(B)}\left(\int_{B} e^{\beta y_{s}} E\left(e^{\beta U_{s}}\right)^{n-1} \mathrm{~d} s\right) .
$$


Then, dominated convergence gives

$$
\begin{aligned}
\lim _{x \rightarrow \infty} & \frac{P\left(X^{1} \in \Gamma\left(\left(x-y_{t}\right)_{t \in T}\right)\right)}{\nu_{1}\left(\Gamma\left(x-\sup _{t} y_{t}\right)\right)} \\
& =e^{-\nu(A)} \frac{1}{e^{\beta \sup _{t} y_{t}} m_{d}(B)} \sum_{n=1}^{\infty} \frac{\nu(A)^{n}}{n !} n\left(\int_{B} e^{\beta y_{s}} E\left(e^{\beta U_{s}}\right)^{n-1} \mathrm{~d} s\right) \\
& =\nu(A) \frac{1}{e^{\beta \sup _{t} y_{t}} m_{d}(B)} \int_{B} e^{\beta y_{s}} \exp \left[\nu(A)\left(E\left(e^{\beta U_{s}}\right)-1\right)\right] \mathrm{d} s \\
& =\nu(A) \frac{\int_{B} e^{\beta y_{s}} E\left(e^{\beta X_{s}^{1}}\right) \mathrm{d} s}{e^{\beta \sup _{t} y_{t}} m_{d}(B)},
\end{aligned}
$$

which with a final reference to Theorem 3.1 and the definition of $\nu_{1}$ concludes the proof.

\section{The main theorem}

Recall that we can write the field $\left(X_{t}\right)_{t \in T}$ as

$$
X_{t}=X_{t}^{1}+X_{t}^{2}+X_{t}^{3}
$$

where the fields $\left(X_{t}^{1}\right)_{t \in T},\left(X_{t}^{2}\right)_{t \in T}$ and $\left(X_{t}^{3}\right)_{t \in T}$ are independent, $\left(X_{t}^{1}\right)_{t \in T}$ is a compound Poisson sum of fields with distribution $\nu_{1},\left(X_{t}^{2}\right)_{t \in T}$ is an infinitely divisible field with Lévy measure equal to the restriction of $\nu$ to $A^{c}$, and $\left(X_{t}^{3}\right)_{t \in T}$ is Gaussian obtained as the integral of $f$ with respect to a Gaussian Lévy basis. Each of the fields in the decomposition has a continuous extension to $B$. In Theorem 3.5 of Section 3 the extremal behaviour of $X^{1}$ was determined. In this section we shall investigate the extremal behaviour of $X^{2}$ and $X^{3}$ in order to obtain the main result on $X$, presented in Theorem 4.5 below.

In the following lemmas we will need to restrict $\nu$ to different subsets of $\mathbb{R}^{T}$. For this we introduce some notation with $0<u<v \leq \infty$

$$
\begin{array}{cl}
A_{(-v,-u]}=\left\{x \in \mathbb{R}^{T}:-v<\inf _{t \in T} x_{t} \leq-u\right\} & A_{[u, v)}=\left\{x \in \mathbb{R}^{T}: u \leq \sup _{t \in T} x_{t}<v\right\} \\
A_{(-v, 0)}=\left\{x \in \mathbb{R}^{T}:-v<\inf _{t \in T} x_{t}<0\right\} & A_{(0, v)}=\left\{x \in \mathbb{R}^{T}: 0<\sup _{t \in T} x_{t}<v\right\} .
\end{array}
$$

Furthermore, we write $A_{(-u, u)}=A_{(-u, 0)} \cup A_{(0, u)}$. Note that e.g., $A^{c}=A_{(-\infty,-1]} \cup$ $A_{(-1,1)}$ except for a $\nu$ null-set, since for $\nu$ almost all $x \in \mathbb{R}^{T}$ it holds that either $x_{t}>0$ for all $t \in T$ or $x_{t}<0$ for all $t \in T$.

Define $\bar{\nu}$ by $\bar{\nu}=\bar{F} \circ V^{-1}$, where $\bar{F}=\xi m_{d} \otimes \bar{\rho}$. Let $\left(Y_{t}\right)_{t \in B}$ be an infinitely divisible random field with Lévy measure $\bar{\nu}$ and cumulant functions

$$
\log E\left(\exp \left(i \sum_{t} \beta_{t} Y_{t}\right)\right)=\int_{\mathbb{R}^{T}}\left(\exp \left(i \sum_{t} \beta_{t} x_{t}\right)-1-i \sum_{t} \beta_{t} x_{t} 1_{[-1,1]^{T}}(x)\right) \bar{\nu}(\mathrm{d} x),
$$

where $\beta \in \mathbb{R}^{T}$ satisfies $\beta_{t}=0$ for all but finitely many $t \in T$. Note that $\left(Y_{t}\right)_{t \in T}$ is defined as the non-Gaussian part of $\left(X_{t}\right)_{t \in T}$ without the drift term and with $\rho$ replaced by $\bar{\rho}$. We can decompose $Y$ into an independent sum

$$
Y=Y^{-}+Y^{(-1,1)}+Y^{+}
$$


where $Y^{-}, Y^{(-1,1)}$ and $Y^{+}$are infinitely divisible with Lévy measures $\bar{\nu}^{-}, \bar{\nu}_{(-1,1)}$ and $\bar{\nu}^{+}$, that are obtained by restricting $\bar{\nu}$ to $A_{(-\infty,-1]}, A_{(-1,1)}$ and $A$ respectively. Note that $\bar{\nu}^{-}$and $\bar{\nu}^{-}$are finite measures due to Lemma A.3 in the Appendix. Furthermore the three fields have cumulant functions

$$
\begin{aligned}
\log E\left(\exp \left(i \sum_{t} \beta_{t} Y_{t}^{-}\right)\right) & =\int_{\mathbb{R}^{T}}\left(\exp \left(i \sum_{t} \beta_{t} x_{t}\right)-1\right) \bar{\nu}^{-}(\mathrm{d} x) \\
\log E\left(\exp \left(i \sum_{t} \beta_{t} Y_{t}^{(-1,1)}\right)\right) & =\int_{\mathbb{R}^{T}}\left(\exp \left(i \sum_{t} \beta_{t} x_{t}\right)-1-i \sum_{t} \beta_{t} x_{t}\right) \bar{\nu}_{(-1,1)}(\mathrm{d} x) \\
\log E\left(\exp \left(i \sum_{t} \beta_{t} Y_{t}^{+}\right)\right) & =\int_{\mathbb{R}^{T}}\left(\exp \left(i \sum_{t} \beta_{t} x_{t}\right)-1\right) \bar{\nu}^{+}(\mathrm{d} x)
\end{aligned}
$$

Lemma 4.1. Let $Y^{(-1,1)}$ be defined as above. Then

$$
E\left(\exp \left(\gamma \sup _{t}\left|Y_{t}^{(-1,1)}\right|\right)\right)<\infty
$$

for all $\gamma>0$.

Proof. Firstly, we argue that $P\left(\sup _{t}\left|Y_{t}\right|<\infty\right)=1$. For this note that $\bar{\rho}$ can be decomposed $\bar{\rho}=\bar{\rho}^{1}+\bar{\rho}^{2}$, where $\bar{\rho}^{1}(\mathrm{~d} x)=C|x|^{-r} 1_{[-1,1]}(x)+C x^{-4} 1_{[-1,1]^{c}}(x)$ and $\bar{\rho}^{2}=\bar{\rho}-\bar{\rho}^{1}$. Thus we also have the decomposition $\bar{\nu}=\bar{\nu}^{1}+\bar{\nu}^{2}$, where $\bar{\nu}^{i}=\bar{F}^{i} \circ V^{-1}$ with $\bar{F}^{i}=\xi m_{d} \otimes \bar{\rho}^{i}$ for $i=1,2$. From this we obtain that $Y$ has the independent decomposition $Y=Y^{1}+Y^{2}$, where $Y^{i}$ has Lévy measure $\bar{\nu}^{i}, i=1,2$. Since $\int x^{2} \bar{\rho}^{1}(\mathrm{~d} x)<\infty$ there exists a version of $Y^{1}$ with a continuous extension to $B$, see Theorem A.1 in The Appendix. In particular $P\left(\sup _{t}\left|Y_{t}^{1}\right|<\infty\right)=1$. Since $\bar{\rho}^{2}([-1,1])=0$, we furthermore see that $\bar{\nu}^{2}=\bar{\nu}_{(-\infty,-1]}^{2}+\bar{\nu}_{[1, \infty)}^{2}$, so $\bar{\nu}^{2}$ is finite due to Lemma A.3 in the Appendix. Thus $Y^{2}$ is a compound Poisson sum of fields that are almost surely on the form $(z f(|t-s|))_{t \in T}$. In particular also $P\left(\sup _{t}\left|Y_{t}^{2}\right|<\infty\right)=1$.

Now we return to the decomposition $Y=Y^{-}+Y^{(-1,1)}+Y^{+}$. We note that $Y^{-}$and $Y^{+}$have continuous extensions to $B$, since both fields are compound Poisson sums of fields with continuous extensions. In particular $P\left(\sup _{t}\left|Y_{t}^{-}\right|<\infty\right)=$ $P\left(\sup _{t}\left|Y_{t}^{+}\right|<\infty\right)=1$, and thereby also $P\left(\sup _{t}\left|Y_{t}^{(-1,1)}\right|<\infty\right)=1$. Furthermore we observe that $\bar{\nu}^{(-1,1)}\left(\left\{x \in \mathbb{R}^{T}: \sup \left|x_{t}\right| \geq 1\right\}\right)=0$ so according to [25, Lemma 2.2] there exists $\epsilon>0$ such that

$$
E\left(\exp \left(\epsilon \sup _{t}\left|Y_{t}^{(-1,1)}\right|\right)\right)<\infty .
$$

Let $\gamma>0$ be given and choose $n \in \mathbb{N}$ such that $\gamma n^{-1 /(r-1)}<\epsilon$. For ease of notation, write $u=n^{-1 /(r-1)}$. We can rewrite the expression for the cumulant function for 
$Y^{(-1,1)}$ using the notation $\left.f_{0}(s)=\sup _{t \in T} f|t-s|\right)$ and the substitution $z=u z$,

$$
\begin{aligned}
\int_{\mathbb{R}^{T}}\left(\exp \left(i \sum_{t} \beta_{t} x_{t}\right)-1-i \sum_{t} \beta_{t} x_{t} 1_{[-1,1]^{T}}(x)\right) \bar{\nu}_{(-1,1)}(\mathrm{d} x) \\
=\int_{\mathbb{R}^{d}} \int_{-1 / f_{0}(s)}^{1 / f_{0}(s)}\left(\exp \left(i \sum_{t} \beta_{t} z f(|t-s|)\right)-1-i \sum_{t} \beta_{t} z f(|t-s|)\right) \frac{C}{|z|^{r}} \mathrm{~d} z \mathrm{~d} s \\
=u^{r-1} \int_{\mathbb{R}^{d}} \int_{-u / f_{0}(s)}^{u / f_{0}(s)}\left(\exp \left(i \sum_{t} \beta_{t} / u z f(|t-s|)\right)-1\right. \\
\left.\quad-i \sum_{t} \beta_{t} / u z f(|t-s|)\right) \frac{C}{|z|^{r}} \mathrm{~d} z \mathrm{~d} s \\
=u^{r-1} \int_{\mathbb{R}^{T}}\left(\exp \left(i \sum_{t} \beta_{t} / u x_{t}\right)-1-i \sum_{t} \beta_{t} / u x_{t}\right) \bar{\nu}_{(-u, u)} \mathrm{d} s
\end{aligned}
$$

This is the cumulant function for $\left(\frac{1}{u} V_{t}\right)_{t \in T}$, if $V$ is an infinitely divisible random field with Lévy measure $u^{r-1} \bar{\nu}_{(-u, u)}=\frac{1}{n} \bar{\nu}_{(-u, u)}$, where $\bar{\nu}_{(-u, u)}$ is the restriction of $\bar{\nu}$ to $A_{(-u, u)}$. Thus, $\frac{1}{u} V$ and $Y^{(-1,1)}$ are identically distributed, so

$$
E\left(\exp \left(\gamma \sup _{t}\left|V_{t}\right|\right)\right)=E\left(\exp \left(\gamma u \sup _{t}\left|Y_{t}^{(-1,1)}\right|\right)\right)<\infty,
$$

since $\gamma u<\epsilon$. Consider the independent decomposition

$$
Y^{(-1,1)}=Y^{(-1,-u]}+Y^{(-u, u)}+Y^{[u, 1)},
$$

where $Y^{(-1,-u]}, Y^{(-u, u)}$ and $Y^{[u, 1)}$ are infinitely divisible fields with Lévy measures $\bar{\nu}_{(-1,-u]}, \bar{\nu}_{(-u, u)}$ and $\bar{\nu}_{[u, 1)}$ obtained by restricting $\bar{\nu}$ to $A_{(-1,-u]}, A_{(-u, u)}$ and $A_{[u, 1)}$. Since $Y^{(-u, u)}$ has the representation

$$
Y^{(-u, u)}=\sum_{k=1}^{n} V^{k},
$$

where $V^{1}, \ldots, V^{n}$ are independent copies of $V$, it follows from (4.1) that also

$$
E\left(\exp \left(\gamma \sup _{t}\left|Y_{t}^{(-u, u)}\right|\right)\right)<\infty .
$$

Furthermore we have that $Y^{(-1,-u]}$ and $Y^{[u, 1)}$ have similar exponential moments, since e.g., $Y^{[u, 1)}$ has compound Poisson representation

$$
Y_{t}^{[u, 1)}=\sum_{k=0}^{M} U_{t}^{k}-y_{t},
$$

where $M$ is Poisson distributed with parameter $\bar{\nu}\left(A_{[u, 1)}\right)$ and the fields $\left(U_{t}^{k}\right)_{t \in T}$ are independent and identically distributed with distribution $\tilde{\nu}_{[u, 1)}=\bar{\nu}_{[u, 1)} / \bar{\nu}\left(A_{[u, 1)}\right)$. Furthermore $y_{t}=\int x_{t} \bar{\nu}_{[u, 1)}(\mathrm{d} x)$. Note that $\left(U_{t}^{k}\right)_{t \in T}$ is non-negative and bounded from above by 1 , and that for each $t \in T$

$$
0 \leq y_{t}=\int x_{t} \bar{\nu}_{[u, 1)}(\mathrm{d} x) \leq \bar{\nu}_{[u, 1)}\left(\mathbb{R}^{T}\right)=\bar{\nu}\left(A_{[u, 1)}\right)<\infty
$$


due to Lemma A.3 in the Appendix. Then

$$
E\left(\exp \left(\gamma \sup _{t \in T}\left|Y_{t}^{[u, 1)}\right|\right)\right) \leq E \exp (\gamma M) \exp \left(\gamma \bar{\nu}\left(A_{[u, \infty)}\right)\right)=\exp \left(\bar{\nu}\left(A_{[u, \infty)}\right)\left(e^{\gamma}-1\right)\right),
$$

which is finite. It is seen with a similar argument that $E\left(\exp \left(\gamma \sup _{t \in T}\left|Y_{t}^{(-1,-u]}\right|\right)\right)$ is finite. Combining this with (4.2) and (4.3), we conclude that also

$$
E\left(\exp \left(\gamma \sup _{t \in T}\left|Y_{t}^{(-1,1)}\right|\right)\right)<\infty \text {. }
$$

Lemma 4.2. We have for all $\gamma>0$ that $E\left(\exp \left(\gamma \sup _{t} X_{t}^{2}\right)\right)<\infty$ and

$$
\lim _{x \rightarrow \infty} e^{\gamma x} P\left(X^{2} \in \Gamma(x)\right)=0 .
$$

Proof. We can write $X^{2}$ as the independent sum of fields

$$
X_{t}^{2}=Z_{t}^{-}+Z_{t}+a_{t},
$$

where $Z^{-}$and $Z$ are infinitely divisible random fields with cumulant functions

$$
\begin{aligned}
\log E\left(\exp \left(i \sum_{t} \beta_{t} Z_{t}^{-}\right)\right) & =\int_{\mathbb{R}^{T}}\left(\exp \left(i \sum_{t} \beta_{t} x_{t}\right)-1\right) \nu_{(-\infty,-1]}(\mathrm{d} x) \\
\log E\left(\exp \left(i \sum_{t} \beta_{t} Z_{t}\right)\right) & =\int_{\mathbb{R}^{T}}\left(\exp \left(i \sum_{t} \beta_{t} x_{t}\right)-1-i \sum_{t} \beta_{t} x_{t}\right) \nu_{(-1,1)}(\mathrm{d} x)
\end{aligned}
$$

such that $\beta \in \mathbb{R}^{T}$ satisfies $\beta_{t}=0$ for all but finitely many $t \in T$. The Lévy measures $\nu^{-}$and $\nu_{(-1,1)}$ are the restrictions of $\nu$ to $A_{(-\infty,-1]}$ and $A_{(-1,1)}$ respectively.

Recalling that $\left(a_{t}\right)_{t \in T}$ is bounded, it suffices to show that $E\left(\exp \left(\gamma \sup _{t} Z_{t}^{-}\right)\right)<\infty$ and $E\left(\exp \left(\gamma \sup _{t} Z_{t}\right)\right)<\infty$ separately for all $\gamma>0$. Since $\nu^{-}$is finite due to Lemma A.3, we can write the $\left(Z_{t}^{-}\right)_{t \in T}$ field on the compound Poisson form

$$
Z_{t}^{-}=\sum_{j=0}^{N} S_{t}^{j},
$$

where $N$ is Poisson distributed with parameter $\nu\left(A_{(-\infty,-1]}\right)$, the fields $\left(S_{t}^{j}\right)_{t \in T}$ are independent and identically distributed with distribution $\bar{\nu}^{-}=\nu^{-} / \nu\left(A_{(-\infty,-1]}\right)$. For each $j$ we have $\sup _{t} S_{t}^{j} \leq-1$, so we see in particular that $E\left(\exp \left(\gamma \sup _{t} Z_{t}^{-}\right)\right)$is finite for all $\gamma>0$. Note that both $Z^{-}$and $Z$ have continuous extensions to $B$, since $Z^{-}$is a compound Poisson sum of fields with continuous extensions. In particular, $P\left(\sup _{t}\left|Z_{t}\right|<\infty\right)=1$.

Since we have the independent decomposition $Y^{(-1,1)}=Z^{1}+Z^{2}$, where $Z^{1}$ is distributed as $Z$, and $Z^{2}$ is infinitely divisible with Lévy measure $\bar{\nu}_{(-1,1)}-\nu_{(-1,1)}$. Note that $\bar{\nu}_{(-1,1)}-\nu_{(-1,1)}$ is a non-negative measure, since $\bar{\rho}-\rho$ is assumed to be non-negative. Also note that $P\left(\sup _{t}\left|Z_{t}^{2}\right|<\infty\right)=1$, since both $\sup _{t}\left|Y_{t}^{(-1,1)}\right|$ and $\sup _{t}\left|Z_{t}^{1}\right|$ are finite almost surely. Applying Tonelli yields

$$
E\left(\exp \left(\gamma \sup _{t} Z_{t}^{1}\right)\right) E\left(\exp \left(\gamma \inf _{t} Z_{t}^{2}\right)\right) \leq E\left(\exp \left(\gamma \sup _{t} Y_{t}^{(-1,1)}\right)\right)
$$

which is finite because of Lemma 4.1. Since the second factor above is clearly nonzero, we conclude that $E\left(\exp \left(\gamma \sup _{t} Z_{t}\right)\right)<\infty$ as desired. 
Lemma 4.3. For all $\gamma>0$, we have $E\left(\exp \left(\gamma \sup _{t} X_{t}^{3}\right)\right)<\infty$.

Proof. From Theorem 4.1.1 in [4] it follows for any $\eta>0$ and $C>0$ that

$$
P\left(\sup _{t} X_{t}^{3}>x\right) \leq C x^{4+\eta} e^{-x^{2} /\left(2 \sigma_{X}^{2}\right)}
$$

where $\sigma_{X}^{2}=E\left(X_{t}^{3}\right)^{2}$. The result of the lemma follows from this.

Theorem 4.4. It holds that

$$
\lim _{x \rightarrow \infty} \frac{P\left(\sup _{t \in B} X_{t}>x\right)}{\nu(\Gamma(x))}=E \exp \left(\beta X_{t_{0}}\right)
$$

as $x \rightarrow \infty$ with $t_{0} \in B$ arbitrarily chosen.

Proof. Let $\pi_{1}$ be the distribution of $\left(X_{t}^{1}\right)_{t \in T}$ and $\pi_{2}$ be the distribution of $\left(X_{t}^{2}\right)_{t \in T}$. Then, with $\epsilon \in(\beta / \gamma, 1)$ for $\gamma>\beta$,

$$
\begin{aligned}
P\left(X^{1}+X^{2} \in \Gamma\left(\left(x-y_{t}\right)_{t \in T}\right)\right)= & \int_{\left\{z: \forall t z_{t}<\left(x-y_{t}\right) \epsilon\right\}} \pi_{1}\left(\Gamma\left(\left(x-y_{t}-z_{t}\right)_{t \in T}\right)\right) \pi_{2}(\mathrm{~d} z) \\
& +\int_{\left\{z: \exists t z_{t} \geq\left(x-y_{t}\right) \epsilon\right\}} \pi_{1}\left(\Gamma\left(\left(x-y_{t}-z_{t}\right)_{t \in T}\right)\right) \pi_{2}(\mathrm{~d} z) .
\end{aligned}
$$

The second term is bounded from above by

$$
\pi_{2}\left(\left\{z: \exists t z_{t} \geq\left(x-y_{t}\right) \delta\right\}\right) \leq \pi_{2}\left(\Gamma\left(\delta\left(x-\sup _{t} y_{t}\right)\right)\right)=o(\nu(\Gamma(x))),
$$

where we have used Theorem 3.1 and Lemma 4.2 since $\gamma>\frac{\beta}{\epsilon}$. Applying Theorem 3.5 and dominated convergence, we obtain for the first term in (4.5) the following

$$
\begin{aligned}
& \sim \nu(\Gamma(x)) \frac{1}{m_{d}(B)} \iint_{B} e^{\beta\left(y_{s}+z_{s}\right)} E\left(e^{\beta X_{s}^{1}}\right) \mathrm{d} s \pi_{2}(\mathrm{~d} z) \\
& =\nu(\Gamma(x)) \frac{1}{m_{d}(B)} \int_{B} e^{\beta y_{s}} E\left(e^{\beta\left(X_{s}^{1}+X_{s}^{2}\right)}\right) \mathrm{d} s \\
& =\nu(\Gamma(x)) \frac{\int_{B} e^{\beta y_{s}} \mathrm{~d} s}{m_{d}(B)} E\left(\exp \left(\beta\left(X_{t_{0}}^{1}+X_{t_{0}}^{2}\right)\right)\right),
\end{aligned}
$$

where the last equality follows, since the distribution of $X_{s}^{1}+X_{s}^{2}$ is independent of $s \in B$. Repeating the arguments using Lemma 4.3 instead of 4.2 gives

$$
P\left(X^{1}+X^{2}+X^{3} \in \Gamma(x)\right) \sim \nu(\Gamma(x)) E\left(\exp \left(\beta\left(X_{t_{0}}^{1}+X_{t_{0}}^{2}+X_{t_{0}}^{3}\right)\right)\right),
$$

which is the desired result.

The theorem below is the main result of our paper. In the formulation of the theorem, we explicitly state the assumptions under which the limit holds. 
Theorem 4.5. Under the assumptions (2.1)-(2.5) on $M$ and (2.6)-(2.8) on $f$,

$$
\lim _{x \rightarrow \infty} \frac{P\left(\sup _{t \in B} X_{t}>x\right)}{L(x) \exp (-\beta x)}=E \exp \left(\beta X_{t_{0}}\right) \xi m_{d}(B)
$$

as $x \rightarrow \infty$ with $t_{0} \in B$ arbitrarily chosen.

Proof. This follows from Theorem 4.4 and Theorem 3.1.

Example 4.6. We consider a model with a NIG basis with parameters $\alpha=0.8$, $\beta=0.6, \mu=0.1, \delta=0.1$ and an exponential kernel function with parameter $\sigma=0.1$, see Examples 2.2 and 2.3. Furthermore, we let $B=[0,20]^{2}$. The level of these parameters is - after a reparameterisation - similar to the level of the parameters estimated in [18]. In Figure 1, simulations of the probabilities $P\left(\sup _{t \in B} X_{t}>x\right)$ are plotted together with the function

$$
E \exp \left(\beta X_{t_{0}}\right) \xi m_{d}(B) x^{-3 / 2} \exp (-(\alpha-\beta) x) .
$$

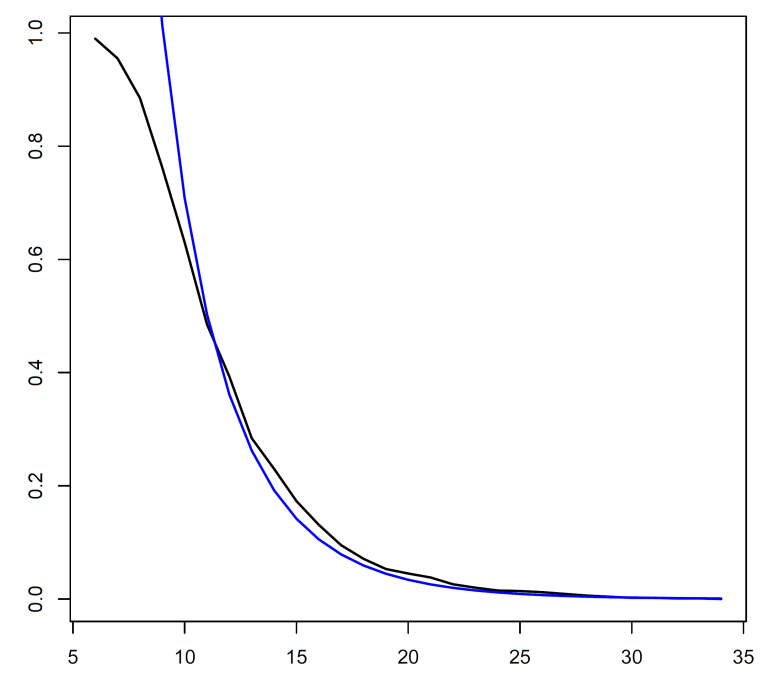

Figure 1: Simulated values of $P\left(\sup _{t \in B} X_{t}>x\right)$ are plotted as a function of $x$ together with the asymptotic theoretical curve in the case of a NIG basis and an exponential kernel function. For details, see text.

\section{$5 \quad$ Excursion sets}

In the present paper, we have been focusing on the asymptotic probability that the supremum of the random field $\left(X_{t}\right)_{t \in B}$ exceeds a level $x$ as $x \rightarrow \infty$. Under the assumptions of our paper, it is also possible to obtain asymptotic results for excursion sets

$$
A_{x}=\left\{t \in B: X_{t} \geq x\right\}, \quad x \in \mathbb{R} .
$$

One example is the asymptotic behaviour of the probability that an excursion set contains a ball of a given size, i.e., the probability of the event

$$
\left\{\exists t_{0} \in B: \inf _{s \in C_{r}\left(t_{0}\right)} X_{s} \geq x\right\},
$$


where $C_{r}\left(t_{0}\right)$ is the ball in $\mathbb{R}^{d}$ with radius $r$ and centre $t_{0}$. Also, this probability is asymptotically described by the right tail of the Lévy measure. The proof is based on the same type of reasoning as in Sections 3 and 4 and is part of a forthcoming paper $([26])$.

\section{Acknowledgements}

This work was supported by Centre for Stochastic Geometry and Advanced Bioimaging, funded by the Villum Foundation.

\section{A Continuous versions of the relevant random fields}

In this Appendix we make the assumptions (2.1)-(2.4) on $M$ and (2.6)-(2.8) on $f$.

Theorem A.1. There exists a continuous version of $\left(X_{t}\right)_{t \in B}$.

Proof. We shall refer to Theorem 2.1 in [19] (see also [19, Theorem 3.1] that corresponds to the case, where $B$ is one dimensional). Note that Theorem 2.1 requires a separable field, but a separable version can be chosen for all random fields, see e.g., [23]. Let $\tilde{B} \supseteq B$ be a box in $\mathbb{R}^{d}$ containing $B$. With a change of measure (see [22] for the existence of a Lévy-Ito decomposition of $M$ ) we can write $Y_{t}=X_{t}-E X_{t}$ on the form

$$
Y_{t}=\int_{\mathbb{R}^{d} \times \mathbb{R}} x f(|t-s|)[N(\mathrm{~d} s, \mathrm{~d} x)-F(\mathrm{~d} s, \mathrm{~d} x)],
$$

where $N$ is a Poisson random measure on $\mathbb{R}^{d} \times \mathbb{R}$ with intensity measure $F$. The integral is well defined since (as is easily verified)

$$
\int_{\mathbb{R}^{d} \times \mathbb{R}}\left((x f(|t-s|))^{2} \wedge|x| f(|t-s|)\right) F(\mathrm{~d} s, \mathrm{~d} x)<\infty
$$

see e.g., $[19$, Section 2] and references therein. Let $D$ be the diameter of $\tilde{B}$. Since $\tilde{B}$ is a box in $\mathbb{R}^{d}$ we have that there exists $a>0$ such that $a m_{d}\left(C_{r}(t) \cap \tilde{B}\right) \geq(r / D)^{d}$ for all $t \in \tilde{B}$ and $r \in(0, D)$. Using the notation from [19] we have

$$
I_{q}\left(a m_{d},|\cdot|, \delta\right)=\sup _{t \in \tilde{B}} \int_{0}^{D}\left(\log \frac{1}{a m_{d}\left(C_{r}(t) \cap \tilde{B}\right)}\right)^{1 / q} \mathrm{~d} r \leq \int_{0}^{D} d(\log D-\log r)^{1 / q} \mathrm{~d} r
$$

which is finite for all $q \geq 1, \delta \in(0, D]$ and in particular for $q=2$ and $\delta=D$. Furthermore we see that $\lim _{\delta \downarrow 0} I_{q}\left(a m_{d},|\cdot|, \delta\right)=0$. From (2.8) and the mean value theorem we find constants $C_{1}, C_{2}>0$ such that

$$
\sup _{0<h \leq D} \frac{|f(x+h)-f(x)|}{h} \leq \frac{C_{1}}{\left(x+C_{2}\right)^{d}}
$$


for all $x>0$. Thus with $g(t,(s, x))=x f(|t-s|)$ (recalling the inequality (2.7) we can find

$$
\|g\|(s, x)=|x|\left(D^{-1} f(|s|)+\sup _{t, u \in \tilde{B}, t \neq u} \frac{|f(|t-s|)-f(|u-s|)|}{|t-u|}\right) \leq|x| \frac{K_{3}}{\left(|s|+K_{4}\right)^{d}}
$$

for some constants $K_{3}, K_{4} \geq 1$ such that for all $c \in(0,1]$

$$
\begin{aligned}
c^{2} F( & \{(s, x):\|g\| \wedge 1>c\}) \leq c^{2} F\left(\left\{(s, x):|x| \frac{K_{3}}{\left(|s|+K_{4}\right)^{d}} \wedge 1>c\right\}\right) \\
& \leq c^{2} F\left(\left\{(s, x): \frac{|x| K_{3}}{c}>|s|^{d},|x|>c / K_{3}\right\}\right) \\
& =c^{2} \int_{\left[-c / K_{3}, c / K_{3}\right]^{c}} m_{d}\left(C_{\left.\left(K_{3}|x| / c\right)^{1 / d}(0)\right) \rho(\mathrm{d} x)}|x|\left(c / K_{3}\right) \rho(\mathrm{d} x) \leq \int_{0}^{\infty} x^{2} \rho(\mathrm{d} x)<\infty .\right.
\end{aligned}
$$

Furthermore, $f$ is bounded and continuous so it follows from [19, Theorem 2.1] that $\left(Y_{t}\right)_{t \in \tilde{B}}$ has a version with continuous sample paths. In particular $\left(X_{t}\right)_{t \in B}$ has a continuous version.

Theorem A.2. There exists a continuous version of the field $\left(X_{t}^{3}\right)_{t \in B}$.

Proof. Obviously, it suffices to find a continuous version of $\left(Y_{t}\right)_{t \in B}$, where $Y_{t}=$ $X_{t}^{3}-E X_{t}^{3}$. The field $\left(Y_{t}\right)_{t \in B}$ has covariance function $\operatorname{Cov}\left(X_{t_{1}}, X_{t_{2}}\right)=R\left(\left|t_{2}-t_{1}\right|\right)$, where for some constant $V$,

$$
R(t)=V \int_{\mathbb{R}^{d}} f(|s|) f(|t+s|) \mathrm{d} s,
$$

see e.g., Section 2 in [18]. The sum $s+t$ is interpreted as adding a vector of length $t$ and fixed direction to $s$. We note that $R$ is continuous and furthermore

$$
R(0)-R(t) \leq V \int_{\mathbb{R}^{d}} f(|s|)|f(|t+s|)-f(|s|)| \mathrm{d} s \leq V C^{\prime}|t| \int_{\mathbb{R}^{d}} f(|s|) \mathrm{d} s,
$$

where we have used the mean value theorem to obtain

$$
|f(|t+s|)-f(|s|)|=\left|f^{\prime}(\xi)\right||| t+s|-| s|| \leq C^{\prime}|t|
$$

with $\xi \in(|s| \wedge|t+s|,|s| \vee|t+s|)$ and $C^{\prime}$ an upper bound for $f^{\prime}$ chosen according to (2.8). In particular for given $\epsilon>0$ there exists $C>0$ such that

$$
R(0)-R(t) \leq \frac{C}{|\log (t)|^{1+\epsilon}}
$$

for all $t>0$ smaller than the diameter of $B$. The existence of a continuous version of $\left(Y_{t}\right)_{t \in B}$ now follows from a corollary to [1, Theorem 3.4.1].

Define for all $\epsilon>0$ the subsets of $\mathbb{R}^{T}$

$$
A_{\epsilon}=\left\{x \in \mathbb{R}^{T}: \sup x_{t} \geq \epsilon\right\} \quad \text { and } \quad B_{\epsilon}=\left\{x \in \mathbb{R}^{T}: \inf x_{t} \leq-\epsilon\right\}
$$


Lemma A.3. For all $\epsilon>0$ we have $\nu\left(A_{\epsilon}\right)<\infty$ and $\nu\left(B_{\epsilon}\right)<\infty$.

Proof. For $A_{\epsilon}$ we find

$$
\begin{aligned}
\nu\left(A_{\epsilon}\right) & =F\left(\left\{(s, z) \in \mathbb{R}^{d} \times \mathbb{R}: \sup _{t \in T} z f(|t-s| \geq \epsilon\}\right)\right. \\
& =\xi \int_{\mathbb{R}^{d}} \rho\left(\left[\frac{\epsilon}{\sup _{t \in T} f(|t-s|)}, \infty\right)\right) \mathrm{d} s \\
& =\frac{\xi}{\epsilon} \int_{\mathbb{R}^{d}} \sup _{t \in T} f(|t-s|) \int x 1_{\left[\frac{\epsilon}{\sup _{t \in T} f(|t-s|)}, \infty\right)} \rho(\mathrm{d} x) \mathrm{d} s \\
& \leq \frac{\xi}{\epsilon}\left(\int_{\mathbb{R}^{d}} \sup _{t \in T} f(|t-s|) \mathrm{d} s\right)\left(\int_{\epsilon}^{\infty} x \rho(\mathrm{d} x)\right)
\end{aligned}
$$

which is finite due to (2.4) and (2.9). The proof for $\nu\left(B_{\epsilon}\right)<\infty$ is identical.

\section{References}

[1] AdLeR, R. (1981). The Geometry of Random Fields. Whiley, Chichester.

[2] Adler, R., Samorodnitsky, G. and Taylor, J. E. (2010). Excursion sets of three classes of stable random fields. Adv. Appl. Prob. 42 293-318.

[3] Adler, R., Samorodnitsky, G. and Taylor, J. E. (2013). High level excursion set geometry for non-Gaussian infinitely divisible random fields. Ann. Prob. 41 (1) 134-169.

[4] Adler, R. And Taylor, J. E. (2007). Random Fields and Geometry. Springer, New York.

[5] Andrews, G., Askey, R. And Roy, R. (1999). Special Functions. Cambridge University Press, Cambridge.

[6] Barndorff-Nielsen, O. (1997). Normal inverse Gaussian distributions and stochastic volatility modelling. Scand. J. Stat. 24 1-13.

[7] Barndorff-Nielsen, O. (1998). Processes of normal inverse Gaussian type. Finance Stochst. 2 41-68.

[8] Barndorff-Nielsen, O. (2010). Lévy bases and extended subordination. Thiele Research Report. 10-12, Department of Mathematics, Aarhus University.

[9] Barndorff-Nielsen, O. (2011). Stationary infinitely divisible processes. Brazil. Journ. of Prob. and Stat. 25 (3) 294-322.

[10] Barndorff-Nielsen, O. And Schmiegel, J (2004). Lévy based tempospatial modelling with applications to turbulence. Uspekhi Mat. Nauk. 159 63-90. 
[11] Cline, D. B. H. (1986). Convolution tails, product tails and domains of attraction. Prob. Th. Rel. Fields 72 529-557.

[12] Cline, D. B. H. (1987). Convolutions of distributions with exponential and subexponential tails. J. Austral. Math. Soc. 43 347-365.

[13] Fasen, V. (2009). Extremes of Lévy driven mixed MA processes with convolution equivalent distributions. Extremes 12 (3) 265-296.

[14] Guttorp, P. And Gneiting, T. (2006). Studies of the history of probability

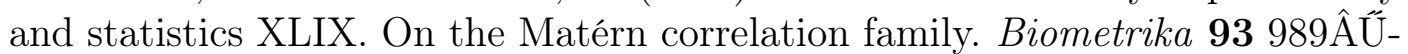
995.

[15] Hashorva, E. AND Ji, L. (2013). Extremes of $\alpha(t)$-locally Stationary Gaussian Random Fields. arXiv.org

[16] Hellmund, G., Prokešová, M And Jensen, E. B. V. (2008). Lévy based Cox point processes. Adv. appl. Prob. 40 603-629.

[17] Jónsdóttir, K. Ý., Schmiegel, J. And Jensen, E.B.V. (2008). Lévy-based growth models. Bernoulli 14(1) 62-90.

[18] Jónsdóttir, K. Ý., Rønn-Nielsen, A., Mouridsen, K. And Jensen, E.B.V. (2013). Lévy-based modelling in brain imaging. Scand. J. Stat. 40(3) $511-529$.

[19] Marcus, M. B. And Rosinksi, J. (2005). Continuity and boundedness of infinitely divisible processes: A Poisson point process approach. J. Theor. Prob. 18 109-ÂÛ́160.

[20] Maruyama, G. (1970). Infinitely divisible processes. Theory Prob. Appl. 15 $1-22$.

[21] Pakes, A. (2004). Convolution equivalence and infinite divisibility. J. Appl. Prob. 41(2) 407-424.

[22] Pedersen, J. (2003). The Lévy Ito decomposition of an independently scattered random measure. MaPhySto Research Report 2003-2.

[23] Potтhoff, J. (2009). Sample properties of random fields. I: separability and measurability. Comm. Stoch. Anal. 3 (1) 143-153.

[24] Rajput, B. S. And Rosinski, J. (1989). Spectral representations of infinitely divisible processes. Prob. Theory Rel. Fields 82 451-488.

[25] Rosiński, J. And Samorodnitsky, G. (1993). Distributions of subadditive functionals of sample paths of infinitely divisible processes. Ann. Probab. 21 996-1014.

[26] Rønn-Nielsen, A. And Jensen, E.B.V. (2014): Excursion sets of infinitely divisible random fields with convolution equivalent Lévy measure. In preparation. 
[27] Sato, K. -I. (1999) Lévy Processes and Infinitely Divisible Distributions. Cambridge University Press. 